\title{
MICCS: A Novel Framework for Medical Image Compression Using Compressive Sensing
}

\author{
Lakshminarayana M. ${ }^{1}$, Mrinal Sarvagya ${ }^{2}$ \\ ${ }^{1}$ Department of ECE, Visvesvaraya Technological University, India \\ ${ }^{2}$ School of ECE, REVA University, India
}

\begin{tabular}{l} 
Article Info \\
\hline Article history: \\
Received Mar 10, 2018 \\
Revised Aug 8, 2018 \\
Accepted Aug 26, 2018 \\
\hline
\end{tabular}

\section{Keyword:}

Compressive sensing

Image compression

Lossless and lossy

compression

Medical image processing

Quality of image

Region of interest

\begin{abstract}
The vision of some particular applications such as robot-guided remote surgery where the image of a patient body will need to be captured by the smart visual sensor and to be sent on a real-time basis through a network of high bandwidth but yet limited. The particular problem considered for the study is to develop a mechanism of a hybrid approach of compression where the Region-of-Interest (ROI) should be compressed with lossless compression techniques and Non-ROI should be compressed with Compressive Sensing (CS) techniques. So the challenge is gaining equal image quality for both ROI and Non-ROI while overcoming optimized dimension reduction by sparsity into Non-ROI. It is essential to retain acceptable visual quality to Non-ROI compressed region to obtain a better reconstructed image. This step could bridge the trade-off between image quality and traffic load. The study outcomes were compared with traditional hybrid compression methods to find that proposed method achieves better compression performance as compared to conventional hybrid compression techniques on the performances parameters e.g. PSNR, MSE, and Compression Ratio.
\end{abstract}

Copyright (C) 2018 Institute of Advanced Engineering and Science. All rights reserved.

Corresponding Author:

Lakshminarayana M., Department of Electronics and communication Engineering, Visvesvaraya Technological University, Belgaum, Karnataka, India.

Email: lakshminarayana.m.2015@ieee.org

\section{INTRODUCTION}

Uttarakhand deluge in India happened on June 2013, has affected destruction comprising breaking of roads, demolition of bridges, restaurants, hospitals, internet connectivity and communication network. As a consequence, about 5700 populates were murdered, and more than 110,000 travelers and tourists stuck in the valleys [1]. This kind of tragedy has strained ever growing responsiveness to refining rescue pains. One of the methods that can efficiently apply throughout tragedy retrieval is recognized as telemedicine in the literature [2] and [3]. Telemedicine is an amalgamation of data computing technology and medical data science. It is utilized to deliver medical data storage and transmission, medical consultation, remote guided robot surgery and many different healthiness care facilities to patients. The convergence of telecommunication technological experts and doctors will provide a platform to encourage next generation medical services. The amount of medical image data that is archived in a hospital is huge, so there is a stringent need to reduce the storage space and transfer time required by these pictures while maintaining the quality of medical image for the diagnostic purpose at the same time. Thus therapeutic image compression productions a significant part for the efficient deployment of next generation healthcare system [4].

Compressed sampling has witnessed a significant attention newly huge courtesy demand for fast, efficient and inexpensive in image and signal processing algorithms, solicitations and medical devices. It is a signal processing method for efficient collection and reconstructing an original signal, by analyzing solutions 
to underdetermined linear schemes. It is novel signal analysis technique its objective is to reconstruct compressed signals from a very less number of measurements. CS assumes that signal satisfies sparsity property [5]. Also, CS requires incoherent acquisition procedure to study, analyze signal reconstruction using compressed sensing. It also aims to offer a technology to design and develop a novel empirical and mathematical model for medical image processing applications. Conflicting to traditional Nyquist standard, the compressive sensing model, investment on a discovery of sparse results to underdetermined linear schemes can renovate the picture signals from a very few sample than is likely using Nyquist sampling rate. The issues of the limited amount of samples can happen in numerous situations, e.g. when we have limited bandwidth, limited storage capacity, measurement are more costly or sluggish to capture such as in radiology and medical imaging modules like MRI, CT, PET, etc. In this type of circumstances, CS offers a favorable solution [6]-[8]. In this CS, there are many algorithms there to reconstruct the original signal, and these are mainly subdivided into the convex optimizations as well as greedy pursuit's schemes [9].

In hybrid mode compressed algorithm, where it integrates both the $\ell 1$-norm as well as $\ell 0$-norm algorithm and results better visual perceptional quality of reconstructed images. Analogous kind of work was done by [10] improves the reconstruction quality of image using the block compressive sensing schemes. In [11] analyzed the compressive sensing method and its specific requirements on medical images using deterministic compressed sensing system. Similar kind of work done by [12] simulated a hybrid compression scheme on different types of medical images using conventional compression methods as well as the neural network based back propagation system to achieve better compression rate. The improvement in visual quality, as well as the better compression ratio, is achieves using Huffman encoding as well as discrete wavelet transform by [13]. In [14] presented a log discrete wavelet transform and logarithmic system to increase the quality of the medical image.

The compression images in [15] presented a mixed compression method using both lossy as well as lossless scheme to achieve a good compression rate and better visual perception quality. The work was carried out in [16] has shown where the hybrid technique recovery is utilized for slice MRI images. The outcomes of this scheme give better recovery rate as well as less computation time. A combined optimization system was presented by [17] for medical images along with non-rigid motion model. This project increases the quality of multiple images using compressive sensing. The compression was also carried out by [18] using a lossless scheme to improve the recovered image quality. A similar type of results obtained in [19] using CS method for MRI images. The block matching algorithm and filtering regularization were done using compression method to reconstruct the original image. Therefore, existing schemes where many compression techniques being presented in recent years for evolving up with novel approaches of compressive sensing.

Elawady et al. [20] designed a data compression schemes and also it eliminates the noise over communication wireless channel. Here, the author uses the Gilbert Elliot channel for wireless communication. It improves the quality of the recovered image and improves the bandwidth of the channel. It also improves the efficiently of recovery images. Zabidi et al. [21] presented a fixed point 8x8-multiplier using advanced DCT schemes to enhance the performance and reduces the longest path in pipeline design. It is implemented using VLSI methodology using TSMC techniques. The obtained outputs gives the better compression ratio as well as good quality of $8 \mathrm{k}$ video and also the timing required to compress the timing also reduced. Sulong et al. [22] studied and implemented a compressive sensing technique along with wiener filter to enhance the reconstructed speech signal at the receiving end. Here author uses the novel CS method, it improves the quality of reconstructed data, compression ratio and removes the noise present in the recovered signal. Sahoo et al. [23], presented a novel image compression techniques using dictionary based algorithms vs. sparse techniques. This method uses the intra-frame prediction scheme to compress images. The output of this scheme improves the overall efficiency of the quality of image as well as the compression ratio of the entire process.

All the methods discussed have a significant favorable point of study and approve while related with restrictions and constraints too. The issues relating to current studies are discussed concisely in the following segment. In this manuscript, we propose a novel framework for medical image compression using hybrid compressive sensing techniques [MICCS]. The new contributions of this proposed paper are, (i) We presented CS based hybrid medical image compression and reconstruction method. We express the reconstructed image and limited bandwidth as a CS problem and then estimate the quality of the reconstructed image of the resulting underdetermined system. (ii) We also proposed a hybrid compressive sensing scheme and it is applied for N-ROI part of a medical image; it successfully incorporates both $\ell 0$ norm and $\ell 1$-normalization of medical image gradient employing presenting a threshold to achieve better image quality. (iii) We also proposed a hybrid version of lossless image compression scheme and it is applied for ROI part of a medical image; it successfully incorporates both Arithmetic and Huffman encoding to achieve better image quality. We also proposed a modified version of hybrid compression recovery algorithm to reduce the necessary computational cost. The simulation results show that using this proposed scheme can 
achieve a better visual perception of an image and lower computational cost compared to other conventional compression methods.

The proposed paper introduces the mixture of both lossless and lossy compression method to achieve better compression rate as well as a good visual perception of reconstructed images. It uses the traditional lossless algorithms are applied to the region of interest part of a picture, and the reaming portion is compressed by using compressive sensing method. It overcomes the optimized dimensional reduction by sparsity into NROI and hence, medical image transmission over limited bandwidth network.

The rest of this paper is organized as follows. Section II discusses the background of compression sensing algorithm. Section III explains about the brief highlights of problem identification. Section IV talked about the proposed technique. Part V took after by discourse of adopted research methodology. Section VI gives the algorithm implementation of the novel algorithm. Section VII continued by comparative performance analysis of the acquired outcomes. At last, the commitments of paper will give the future research direction is summarized in Section VIII.

\section{BACKGROUND}

The concepts of CS has been fruitfully applied to increase the speed of medical image reconstruction with Fourier encoding method. The total variation (TV) is frequently utilized as the normalization function for medical image reconstruction. But, it is recognized that such $l_{1}$-based normalization scheme more essentials than $l_{0}$-based technique. But in another side, $l_{0}$-based normalization is computational inflexible and unstable. So we proposed a novel hybrid method, which effectively integrates both $l_{1}$ and $l_{0}$-norm of image gradient by applying a threshold [24]. The HTV normalization scheme has more benefits of both robustness of $l_{1}$ and very fewer measurements of $l_{0}$.

Precisely, CS deliberates the issue to recovery a signal $x$ of size $n$ from m linear measurements $y=\Phi x$. Where $m<<n$. So, like that, an underdetermined scheme involves the infinite value of solutions in general. Along with the previous data, the sign $x$ is sparse; the most scattered result must be sought. Initially, $l_{1}$ is a better metric for the sparsity of signal. The data recovered by resolving forced $l_{0}$ normalization issues,

$$
\min _{x}\|x\|_{0} \quad \text { s.t. } \Phi x=y
$$

where $\|x\|_{0}=\sum_{i} I_{t}, \quad I_{t}= \begin{cases}1, & x_{t} \neq 0 \\ 0, & x_{t}=0\end{cases}$

Still, the problem of $l_{0}$ is computationally inflexible and unstable. The very populate surrogate for $l_{0}$ and $l_{1}$ definition as,

$$
\|x\|_{i}=\sum_{t}\left|x_{t}\right|
$$

It is main because $l_{1}$-norm method is convex and also the robustness of $l_{1}$ normalization is guaranteed [25]. But additional measurements are mandatory for $l_{0}$ minimization [26]. Non-convex substitutes take also stood studied [27] to minimize the sizes of $l_{1}$, nevertheless typically at the cost of improved computational complexity and decreased robustness. CS has authenticated achievement in speed up MRI via small sampling [28]. The measurement is prepared in $k$-space or spatial domain. In CS recovery, TV or $l_{1}$-norm of medical image gradient is typically used in constrained standardization [29],[30]. Thus, TV is defined as,

$$
\|x\|_{T V}=\sum_{i, j}\left|\Delta x_{i, j}\right|=\sum_{i, j} \sqrt{\left(D_{i, j}^{h} x\right)^{2}+\left(D_{i, j}^{v} x\right)^{2}}
$$

where, $D_{i, j}^{h} x$ and $D_{i, j}^{v} x$ are the parallel and perpendicular slopes of an image at position $(i, j)$. TV takes the pleasant assets of convex and strength. Though, the necessary amount of $k$-space models is huge while the image has various parts.

\section{PROBLEM IDENTIFICATION}

This section deals with the issues identified after reviewing various existing research works related to the hybrid medical image compression using compressive sensing. A closer look towards the current compressive sensing techniques is utilized to achieve more compression on critical image regions, even knowing that it is lossy compression method. Hence, the current usage of CS is not for analysis of various 
complex medical images and also to achieve faster response time. The delay in current research works address lesser quality of the image as well as large algorithm processing time. These issues are addressed in proposed work. All the existing works achieve image compression has a certain limit, but it also some specific foremost loopholes presents. As the outcome of image analysis cannot be associated with errorproneness, hence more emphasis is formulated over the open research problems. Some of limitation or issues of medical image compression are discussed here are:

a. In the existing work more are less everyone uses the conventional image compression methods to compress the images. But medical image analysis is suffering from storage and transmission as well as limited bandwidth etc. Less efficient techniques are found in current literature work toward hybridizing schemes in innovative directions. It will decreases the image cost, reduces the dimension of image and improves the quality of images.

b. Accurate diagnosis of illnesses and abnormality circumstance needs both region-of-interest and nonregion-of-interest portion of recovered medical image with less cost and less time. Unluckily, the current research works are not focusing on non-region part of image and not found. So this point considers this fact to carry out of medical image compression and reconstruction using hybrid technology.

c. We reviewed various existing methods, these techniques achieves greater compression rate on various medical images. But the pitfalls of the current system are, i) assessing the similar type of dataset, ii) there is no comparative study and iii) image analysis uses less number of performance parameters etc. It will suggest towards future research direction. i.e, very less amount of studies towards standard medical image compression.

Less number of studies towards image reconstruction to achieve good quality of reconstructed image as well as greater dimensionality reduction on both ROI part and Non-ROI part of image.

\section{PROPOSED METHOD}

The proposed work is a continuation of our previous studies [31]-[37]. Where compression of medical image has been refined to get better compression performance. This part of study especially focused on the region based medical image while performing a novel compression technique. We present a novel framework for Medical Image Compression using Compressive Sensing [MICCS] in this implementation paper in the direction to improve out previous work towards increasing better compression on both diagnostically important region as well as the non-diagnostically region. The design of a novel framework of MICCS is shown in Figure 1. Where we used the different medical images as inputs for analyzing our proposed scheme.

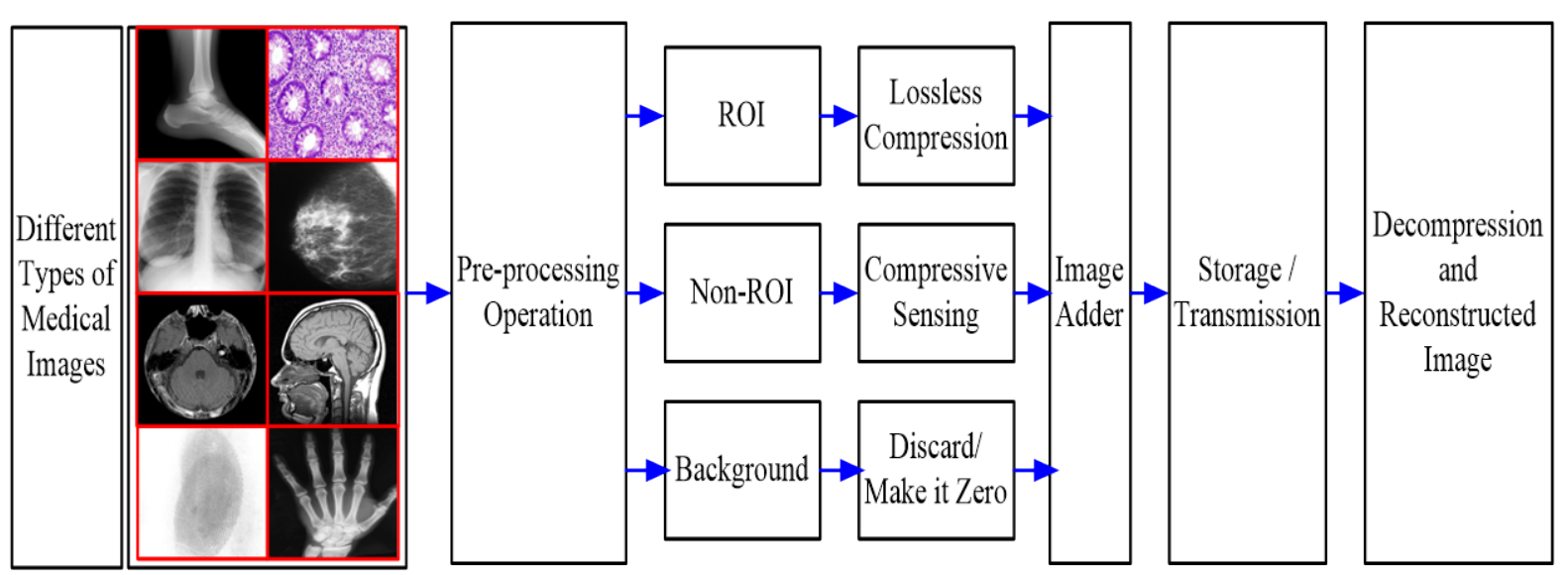

Figure 1. Architecture of the proposed MICCS method

In this scheme, firstly, we will collect all the input images in a database. Then applying the preprocessing operations to all the medical images. Then, the image is divided into three different part: i) the background of an image, ii) the region of interest of the image, and iii) the non-region of focus part. The background of an image is discarded to reduce the size of the image. The central part is ROI, i.e. diagnostically important region is compressed with the tradition method like Huffman and arithmetic encoding schemes to achieve better image quality of ROI part. Then final the compressive sensing algorithm 
is applied to N-ROI part. It also achieves better reconstruction of an image compared to traditional schemes. The simulated system reduces the HTV of chosen image x exposed to the reliable information limitation. Without the cost of simplification, assume the limited slope $\Delta_{i, j}^{x}$ is constrained by one through the full image. Scientifically, we resolve,

$$
\min _{x}\|x\|_{H T V} \quad \text { s.t. } \Phi x=y
$$

where as $\Phi$ is Fourier coding matrix for minimized sampling, $y$ is the under sampled $k$-space information, HTV is well-defined as,

$$
\|x\|_{H T V}=\sum_{i, j} f\left(\Delta_{i, j} x\right)
$$

where $f\left(\Delta_{i, j} x\right)=\left\{\begin{array}{r}\left|\Delta_{i, j} x\right|,\left|\Delta_{i, j} x\right|<\tau \\ 1,\left|\Delta_{i, j} x\right| \geq \tau\end{array}\right.$ as well as $\tau \leq 1$ is a stable threshold. It is shown that the projected HTV comprises TV as a clear case when $\tau=1$. Such as $\tau$ reaches zero, HTV converts the $l_{0}$ of the image ascent. In place of any $0<\tau<1$, HTV fusions $l_{1}$ and $l_{0}$ reliant on the natural gradient at separate places and be able to be viewed as a mixture $l_{1}-l_{0}$ of the image ascent. The cost of $\tau$ controls the assistances on or after $l_{1}$ or $l_{0}$ correspondingly [23]. For large $\tau$, HTV is quicker to a convex task and therefore consumes improved convergence to the universal minimum. We have demonstrated, we resolve the unrestrained optimization issues in its place:

$$
\left(\min _{x}\|y-\Phi x\|_{2}+\lambda\|x\|_{H T V}\right)
$$

where the regularization or normalization parameters $\lambda$ is selected to stability the impact of each duration. Gradient descent technique [12] is recycled to an appliance of the projected HTV minimization process. In every repetition, the image at pixel (i, j) is restructured by,

$$
x_{i, j}^{k+1}=x_{i, j}^{k}-\mu_{j}^{k} \Delta_{i, j} H\left(X^{k}\right)
$$

where as $\mathrm{H}\left(X^{k}\right)$ is the objective function well-defined in Eq. (7), $k$ signifies the repetition index, and $\mu$ is the gradient background issue. The grade of the HTV is assumed as the quantity of,

$$
\Delta_{i, j}\|x\|_{H T V}=\left\{\frac{D_{i, j}^{h} x}{\left|\nabla x_{i, j}\right|}+\frac{D_{i, j}^{v} x}{\left|\nabla x_{i, j}\right|}-\frac{D_{i-1, j}^{h} x}{\left|\nabla x_{i-1, j}\right|}-\frac{D_{i, j-1}^{v} x}{\left|\nabla x_{i, j-1}\right|}\right.
$$

where $\left|\nabla x_{i, j}\right|<\tau$, above entire pixels, where a minor $\varepsilon$ is extra to the denominator to escape separating by zero. After the similar optimization algorithm (e.g. gradient descent) is utilized, the computational difficulty of the projected technique is around the similar as that of TV. But, due to the non-convexity of HTV, a correct early estimate is needed for the conjunction to a great superiority image [24]. In our training, we habit the TV renovation as the original estimate for the picture.

The core objectives of proposed study are i) To present a non-iterative technique for compressing a radiological image, ii) To introduce a modeling of a combinatorial approach to accomplish higher compression performance. MICCS is designed on the concept of implementing lossy compression scheme of compressive sensing on non-ROI while it applies lossless compression schemes on ROI portion of the image. This methodology is undertaken to ensure a better equilibrium between compression performance and image quality of output signal. Next section discusses the research methodology being adopted to execute the MICCS.

\section{RESEARCH METHODOLOGY}

The execution of the proposed work is conducted, considering an analytical research methodology. The procedure for implementing the proposed work has explained below:

\subsection{Selecting the ROI and Non-ROI part of Image}

In this section, we considered various classes of medical images from a dataset to achieve better result assessment. Here, the input image is converted into a grayscale image. The proposed MICCS technique considers compression an input image in region based methods. The ROI part of an image is compressed 
with the traditional lossless compression method and the Non-ROI part of the image is compressed with Compressive Sensing method. The background of an image is discarded. These regions are divided based on segmentation algorithm. The ROI is confirmed using bounding box and also the non-ROI is retained as it is in this part in Figure 2.

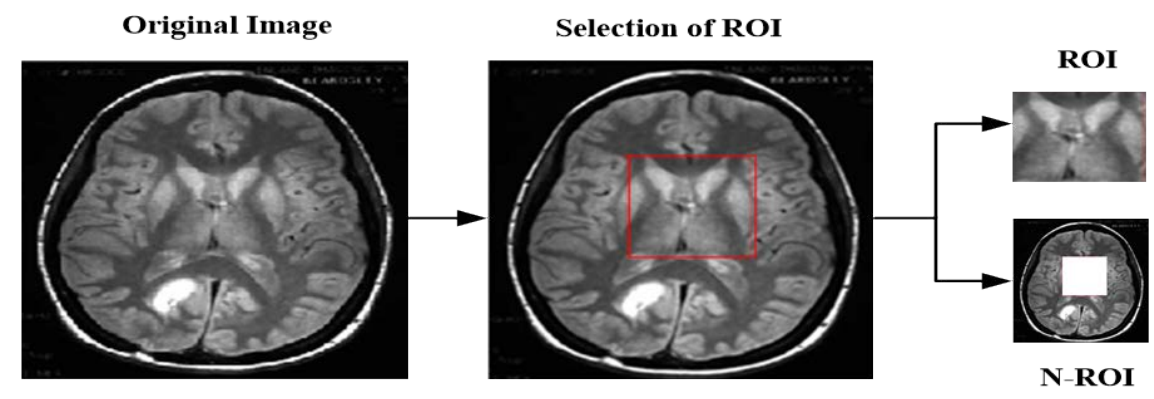

Figure 2. Preparing the input image

\subsection{Hybrid Compression of Image}

After selection of ROI and non-ROI of an image, the work is to execute the compression on a specific range of compression. The ROI part of the image is diagnostically important for doctors to detect the diseases of the patient. So, this part of an image is compressed with the traditional lossless compression techniques (such as JPEG 200, Huffman coding and Run-length encoding) to achieve a good quality of the reconstructed image. Then, the non-ROI part of an image is considered as an important part, so our work highlights non-ROI potion and hence it is compressed with lossy compression scheme like compressive sensing algorithm in Figure 3. For this, we initialized some basic parameters like size of the coefficient for compressive sensing.

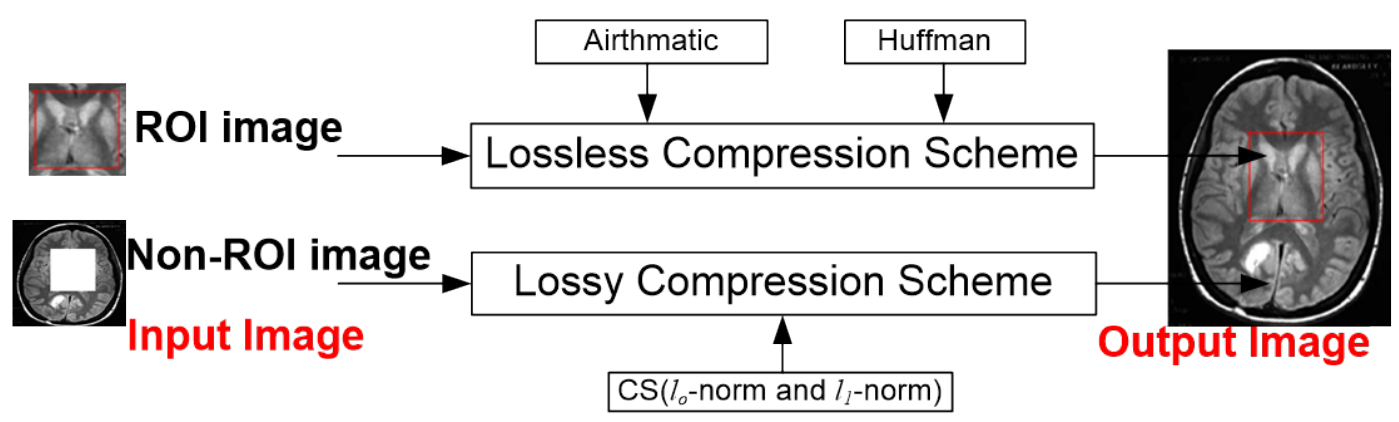

Figure 3. Performing hybrid compression scheme

\section{ALGORITHM IMPLEMENTATION}

The design of proposed algorithm (MICCS) is conducted in three different stages i.e., the preparation of medical images, image preprocessing, dividing the ROI, non-ROI part of image etc. Then, it is followed by dimensionality reduction of images using hybrid compression of lossless for ROI and compressive sensing for non-ROI part of images. Then, finally performing the decompression as well as reconstruction of medical image.

\subsection{Proposed Hybrid Algorithm (MICCS):}

Input: $I_{1}$ (ROI), $I_{2}$ (NROI), L (length of Coefficient), $m$ (number of rows), $n$ (number of columns), $\delta$ (n-size observation vector), $\psi(n \times p)$ measurement matrix;

Initialized input: $S$ (sparsity level), L (number of taps);

Output: $I_{3}$ (Reconstructed image); 


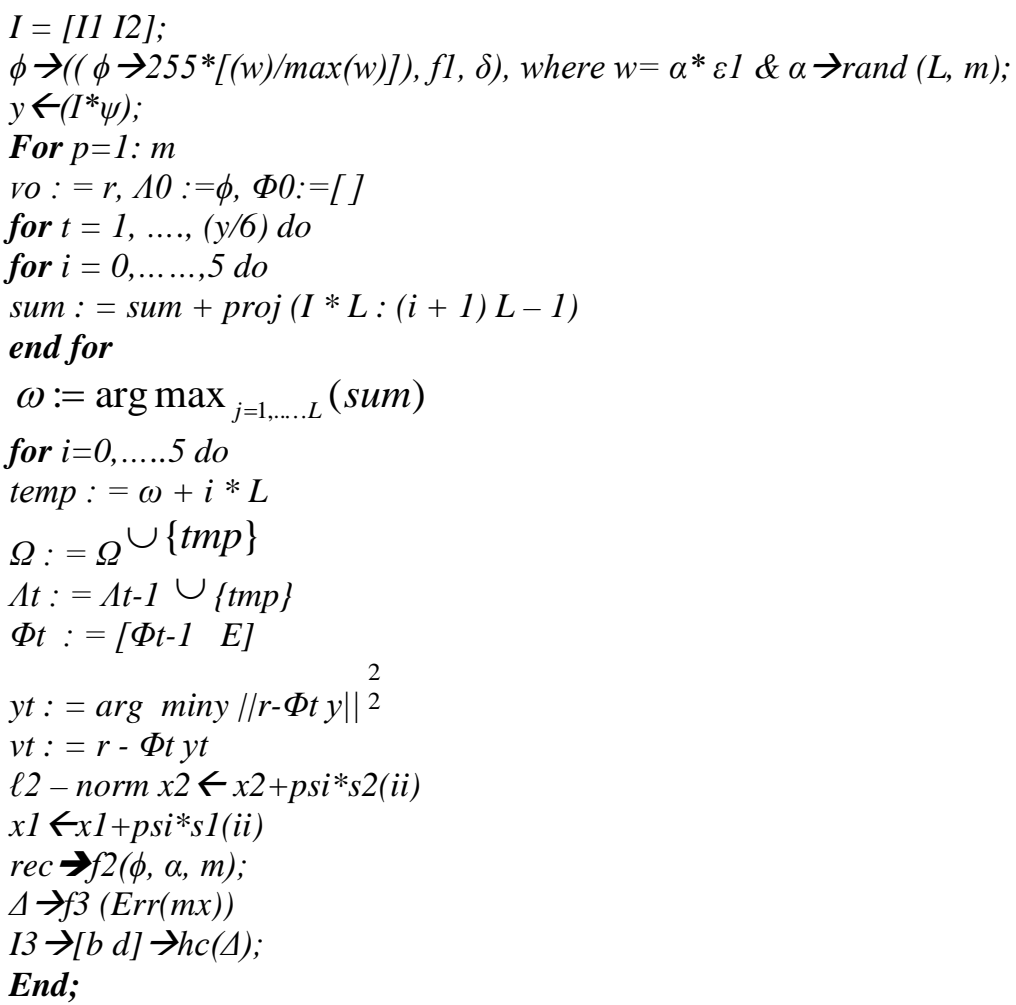

The proposed MICCS algorithm deliberate input of $I_{1}$ (region of interest) and $I_{2}$ (non-region of interest), $L$ (measurement of the coefficient for CS), $m$ number of rows, $n$ number of columns, $\delta$ is range of compression ratio. The system outlines length of coefficient $(L)$ value as 150 for CS that is monitored by means of a new element $\sigma$. This characteristic chooses the coefficient arbitrarily on the base of rows $m$. An aspect $\phi$ is produced by convolution of $\alpha$ and $\varepsilon 1$, where $\left.\epsilon_{1}=\sum[\theta(I) \cdot \psi \cdot \psi T\rceil T\right]$. The factor $\psi$ is a 2D measurement that is correspondent to $m$ rows, although $\theta$ is sparse matrix of image. This method will produce definitive consequence of $\phi$.

Parameters initialization will be done using unsigned integers of 8-bits from $\phi$ as well as implementing JPEG2000 standard and compression ratio $\delta$. An element of $\phi$ is yet produced once again by produced bits as healthy as exploiting its accuracy to double. Finally, the reconstructed image rec is designed using hybrid image compression of both lossless at the ROI part and lossy-CS at non-ROI part of input image. ROI coding is formerly originated by assessing error Err. The parameter $m x$ represents the noniterative coefficients in an image. The final consequential is applied for subjecting to following procedure of run-length coding to achieve $\Delta$ (data) conveyed by Huffman encoding in direction to improve superiority of radiological image. In the procedure $f 1, f 2, f 3$ is a purpose used for JPEG2000, Compressive Sensing and Run-Length coding respectively. The significant object to comprehend is ROI image was exposed to lossless compression technique, whereas non-ROI image existed exposed to lossy compression method of CS. The subsequent segment will discuss about the results obtained from the proposed work.

\section{RESULTS AND ANALYSIS}

In this section, we demonstrate an efficient hybrid medical image compression using both lossless and compressive sensing algorithm. We compared, our projected technique with the another traditional hybrid compression method to estimate its performance. Quantitative assessment and comprehensive investigation of the investigational consequences are obtainable in each section, and constraints setting are assumed beforehand every experimentation.

The evaluation of the proposed work uses standard medical datasets of Cornell University [38]. It studies more than 1000 medical images of different types. We implementing a novel MICSS algorithm using hybrid lossless (JPEG-2000+Huffman + Run length) and also hybrid of CS ( $l_{0}$-norm $+l_{1}$-norm) algorithm for ROI and non-ROI part of images respective, and also achieves good quality of reconstructed image. Table 1 highlights the Original image, ROI, Non-ROI and Reconstructed image results of the test medical images obtained in proposed method. Table 2 demonstrates the measurement matrix of the selected signal along with the compressed, normalized and sparsity signal. 
Table 1. Original image, ROI, non-ROI and Reconstructed Medical images for proposed work (MICCS)

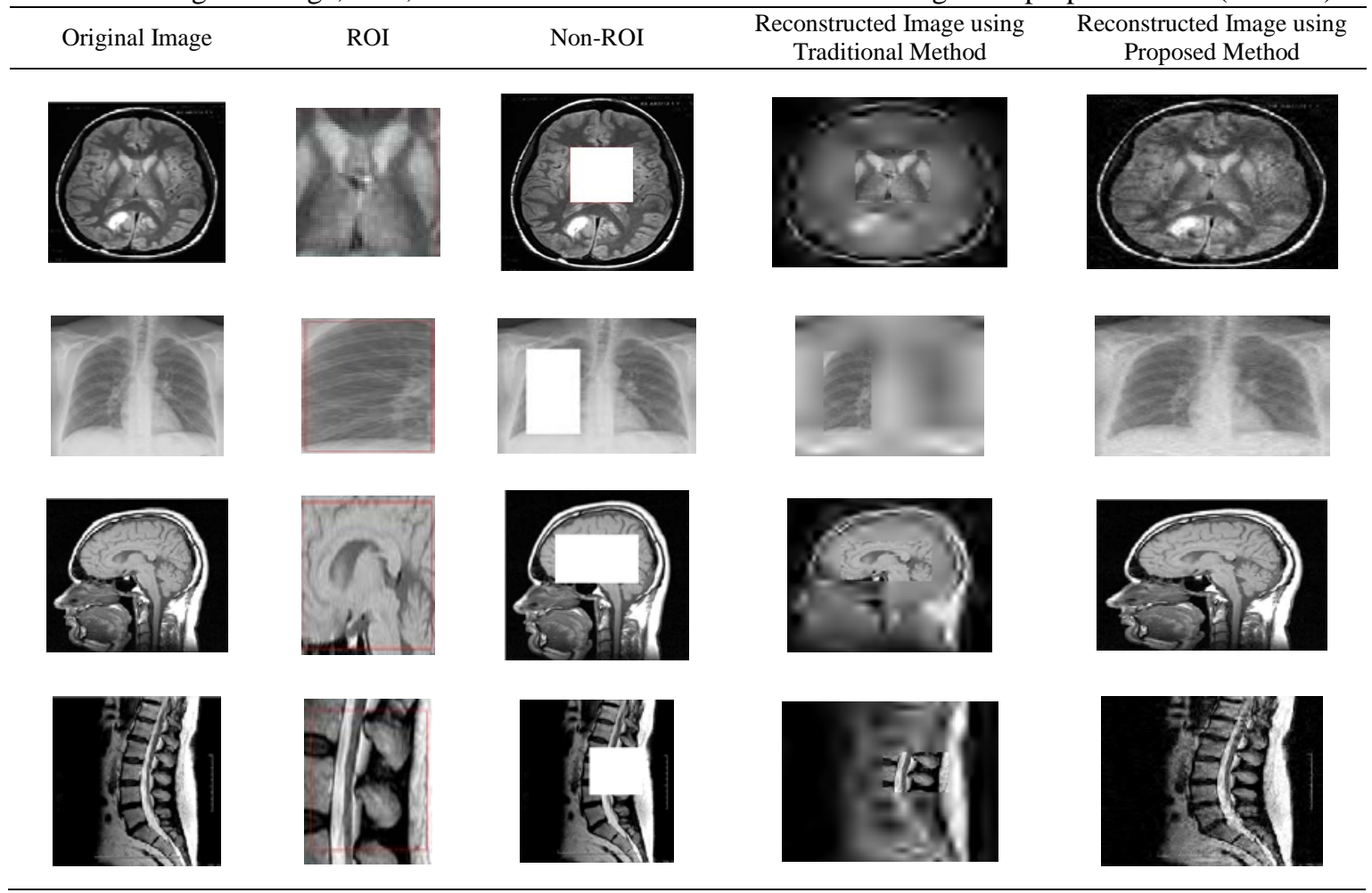

Table 2. Demonstrates Measurement Matrix, Compressed Signal

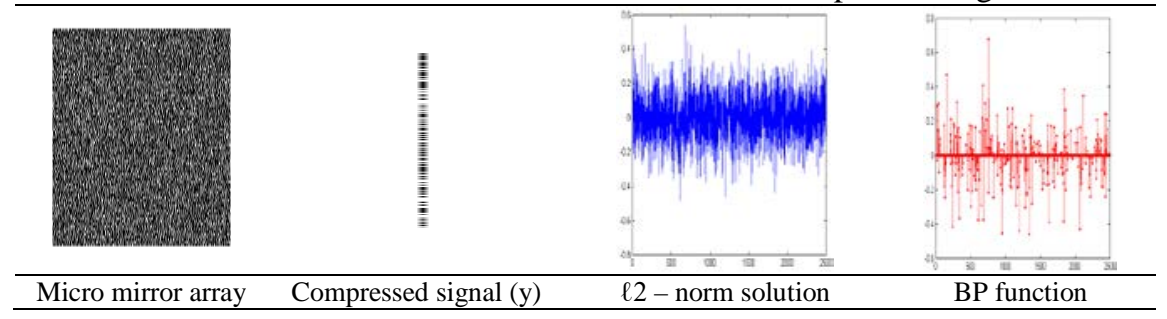

Figure 4 Illustrates the normalized signal. Table 3 highlighted the ROI and Reconstructed full image results of the test medical images obtained in proposed method. The proposed system makes a minor amendment to the existing system to create a similar test-bench to carrying out comparative analysis considering the performance parameter of PSNR, MSE and Compression ratio.

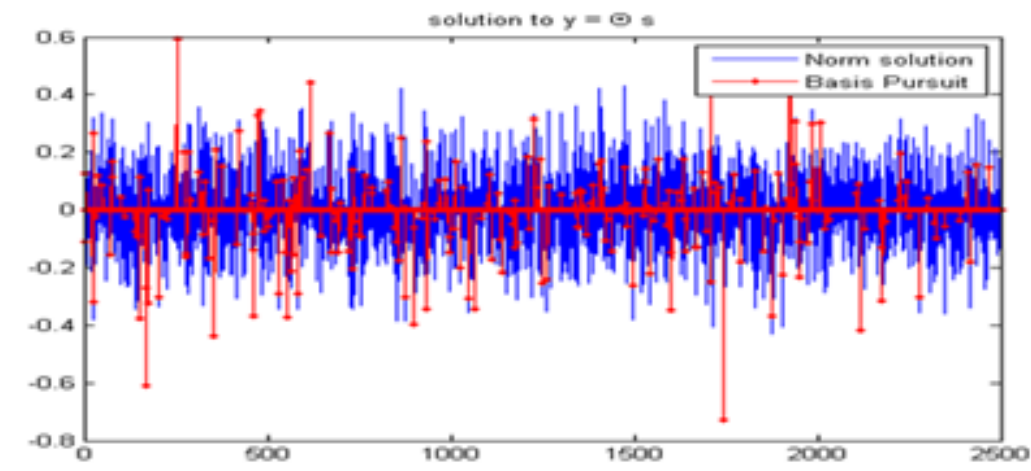

Figure 4. Normalized Signal 
Table 3 indicates the modifications done to ensemble the present method to be compared with projected combinatorial methodology to evaluate the compression presentation. In method-1, we applied Huffman encoding scheme to ROI part of image and SPIHT is applied to non-ROI part of the image. Similarly, in method-2, arithmetic and SOM is applied to ROI and non-ROI part of images respectively. But in our proposed method uses the combination of JPEG2000, RLE and Huffman encoding is used to ROI and Compressive sensing is applied to non-ROI part of an image to achieve the better visual perceptional quality of an image.

Table 3. Comparative Analysis Using Different Hybrid Techniques

\begin{tabular}{ccc}
\hline Scheme & ROI & Non-ROI \\
\hline Method-1 & Huffman & SPIHT \\
Method-2 & Arithmetic & Self-Organizing Map \\
Proposed (MICCS) & JPEG2000, RLE, Huffman & Compressive Sensing \\
\hline
\end{tabular}

We tested seven different bits per pixel (bpp) for medical images. All the images are reconstruction using the traditional hybrid compressed method and proposed methods. The experimental results evaluated by PSNR, MSE and CR are given in Table 4 to 6 . Generally speaking, the greater the sampling rate, the more prior information the compressive sample contains, and then the medical image can be reconstructed with higher accuracy. The experimental results verify this regularity. The value of the PSNR of the reconstruction signal results increases along with the sampling rates.

Table 4. ROI image and full image analysis (MICCS) for PSNR, MSR and CR

\begin{tabular}{cccccccc}
\hline Images & BPP & PSNR & $\begin{array}{c}\text { ROI Region } \\
\text { MSE }\end{array}$ & CR & PSNR & $\begin{array}{c}\text { FULL Image } \\
\text { MSE }\end{array}$ & CR \\
\hline Image-1 & 1.000 & 41.484 & 8.596 & 5.512 & 34.922 & 208.796 & 11.084 \\
Image-2 & 0.500 & 39.995 & 14.942 & 8.985 & 32.885 & 219.955 & 21.933 \\
Image-3 & 0.400 & 38.566 & 16.644 & 12.018 & 31.508 & 226.532 & 24.975 \\
Image-4 & 0.300 & 36.998 & 17.192 & 13.494 & 29.897 & 237.780 & 30.861 \\
Image-5 & 0.250 & 36.012 & 19.878 & 16.046 & 27.479 & 239.814 & 42.425 \\
Image-6 & 0.125 & 35.871 & 21.836 & 19.975 & 25.551 & 321.999 & 125.778 \\
Image-7 & 0.0625 & 35.095 & 22.997 & 21.342 & 24.930 & 389.513 & 252.010 \\
\hline
\end{tabular}

Table 5. ROI image, Non-ROI image and Full Image Analysis (MICCS) for PSNR

\begin{tabular}{ccccc}
\hline Images & BPP & $\begin{array}{c}\text { ROI } \\
\text { PSNR }\end{array}$ & $\begin{array}{c}\text { FULL Image } \\
\text { PSNR }\end{array}$ & $\begin{array}{c}\text { Non-ROI } \\
\text { PSNR }\end{array}$ \\
\hline Image-1 & 1.000 & 41.484 & 34.922 & 33.054 \\
Image-2 & 0.500 & 39.995 & 32.885 & 32.428 \\
Image-3 & 0.400 & 38.566 & 31.508 & 31.96 \\
Image-4 & 0.300 & 36.998 & 29.897 & 30.125 \\
Image-5 & 0.250 & 36.012 & 27.479 & 28.347 \\
Image-6 & 0.125 & 35.871 & 25.551 & 26.982 \\
Image-7 & 0.0625 & 35.095 & 24.930 & 24.356 \\
\hline
\end{tabular}

Table 6. Comparison Results for Different Traditional Hybrid Techniques VS. Proposed Technique (MICCS) in Terms of PSNR, MSE \& CR

\begin{tabular}{cccccccccc}
\hline Images & PSNR & $\begin{array}{c}\text { Method1 } \\
\text { MSE }\end{array}$ & CR & PSNR & $\begin{array}{c}\text { Method2 } \\
\text { MSE }\end{array}$ & CR & PSNR & $\begin{array}{c}\text { Proposed } \\
\text { MSE }\end{array}$ \\
\hline Image-1 & 24.089 & 289.468 & 38.253 & 23.075 & 287.056 & 30.548 & 34.922 & 208.796 \\
Image-2 & 23.456 & 293.154 & 42.998 & 22.587 & 291.578 & 31.048 & 32.885 & 219.955 \\
Image-3 & 23.893 & 298.365 & 48.154 & 20.876 & 290.254 & 33.487 & 31.508 & 226.532 \\
Image-4 & 21.082 & 427.859 & 57.072 & 20.009 & 305.456 & 39.487 & 29.897 & 237.780 & 24.973 \\
Image-5 & 20.361 & 550.256 & 62.287 & 19.879 & 315.478 & 40.587 & 27.479 & 239.814 & 42.425 \\
Image-6 & 19.524 & 590.872 & 66.464 & 17.587 & 420.258 & 59.548 & 25.551 & 321.999 & 125.778 \\
Image-7 & 18.075 & 633.258 & 69.325 & 16.897 & 570.265 & 65.254 & 24.930 & 389.513 & 252.010 \\
\hline
\end{tabular}

\section{CONCLUSION}

The aim of this manuscript is to check the applicability of hybrid compression methods over different medical images. It was achieved that, the compressed sensing scheme offers optimal compression 
rate by using minimum sample measurements. This provides network based efficiency regarding channel bandwidth as well as the storage of a vast number of data transmission to improve the faster response time. A significant research gap was identified towards the reconstruction image using compression sensing methods. The presented work makes the balanced use of lossy and lossless compression schemes which applied to complete radiologic pictures in a discrete manner. The novel MICCS uses both lossy schemes such as hybrid compression sensing algorithms over Non-ROI part of the image; the ROI is compressed with hybrid lossless compression methods employed. The outcomes of the MICCS was found to achieves a superior quality of an image, better compression rate and fewer means square error compared to other compression schemes.

\section{REFERENCES}

[1] Uttarakhand Flood, 2014. http://en.wikipedia.org/wiki/2013_North_India_floods.

[2] B. Fong, et al., "Prognostics and health management for wireless telemedicine networks," IEEE Wireless Commununication, vol/issue: 19(5), pp. 83-89, 2012.

[3] K. Kang, et al., "Design and QoS of a wireless system for real-time remote electrocardiography,” IEEE J. Biomed, Health Inform., vol/issue: 17(3), pp. 745-755, 2013.

[4] J. Tan, et al., "Compressive Hyperspectral Imaging via Approximate Message Passing," in IEEE Journal of Selected Topics in Signal Processing, vol/issue: 10(2), pp. 389-401, 2016.

[5] A. S. Unde, et al., "Low complexity secure encoding and joint decoding for distributed compressive sensing WSNs,” 3rd International Conference on Recent Advances in Information Technology (RAIT), Dhanbad, pp. 89-94, 2016.

[6] M. Azghani, et al., "Multihypothesis Compressed Video Sensing Technique," in IEEE Transactions on Circuits and Systems for Video Technology, vol/issue: 26(4), pp. 627-635, 2016.

[7] Z. Chen, et al., "Reconstruction of Enhanced Ultrasound Images From Compressed Measurements Using Simultaneous Direction Method of Multipliers,” in IEEE Transactions on Ultrasonics, Ferroelectrics, and Frequency Control, vol/issue: 63(10), pp. 1525-1534, 2016.

[8] H. Lee, et al., "A method for co-existing heterogeneous IoT environments based on compressive sensing," 18th International Conference on Advanced Communication Technology (ICACT), Pyeongchang, pp. 206-209, 2016.

[9] J. Sevilla, et al., "Hyperspectral image reconstruction from random projections on GPU," IEEE International Geoscience and Remote Sensing Symposium (IGARSS), Beijing, China, pp. 280-283, 2016.

[10] Ponuma R., et al., "Cosine Number Transform based hybrid image compression-encryption,” International Conference on Wireless Communications, Signal Processing and Networking (WiSPNET), Chennai, pp. 172-176, 2016.

[11] K. Kulkarni and P. Turaga, "Reconstruction-Free Action Inference from Compressive Imagers,” in IEEE Transactions on Pattern Analysis and Machine Intelligence, vol/issue: 38(4), pp. 772-784, 2016.

[12] M. V. R. Manimala, et al., "Sparse recovery algorithms based on dictionary learning for MR image reconstruction," International Conference on Wireless Communications, Signal Processing and Networking (WiSPNET), Chennai, pp. 1354-1360, 2016.

[13] L. Zhang, et al., "Locally Similar Sparsity-Based Hyperspectral Compressive Sensing Using Unmixing," in IEEE Transactions on Computational Imaging, vol/issue: 2(2), pp. 86-100, 2016.

[14] A. M. Ashir and A. Eleyan, "Compressive sensing based facial expression recognition," 24th Signal Processing and Communication Application Conference (SIU), Zonguldak, pp. 1241-1244, 2016.

[15] A. C. Sankaranarayanan, et al., "Enhanced Compressive Imaging Using Model-Based Acquisition: Smarter sampling by incorporating domain knowledge,” in IEEE Signal Processing Magazine, vol/issue: 33(5), pp. 81-94, 2016.

[16] V. Angayarkanni, et al., "Distributed compressive video coding using Enhanced side information for WSN," International Conference on Wireless Communications, Signal Processing and Networking (WiSPNET), Chennai, pp. 1133-1136, 2016.

[17] M. Marić, et al., "Compressive sensing based image processing in trapview pest monitoring system," 39th International Convention on Information and Communication Technology, Electronics and Microelectronics (MIPRO), Opatija, pp. 508-512, 2016.

[18] C. Y. Qiong, et al., "Underwater acoustic image compressive sensing algorithm research based on Bandelets transform,” OCEANS 2016 - Shanghai, Shanghai, pp. 1-5, 2016.

[19] Y. Fu, et al., "Image Compressive Sensing via Multiple Constraints," Eighth International Conference on Measuring Technology and Mechatronics Automation (ICMTMA), Macau, pp. 327-330, 2016.

[20] I. Elawady, et al., "The Noise Reduction over Wireless Channel Using Vector Quantization Compression and Filtering,” International Journal of Electrical and Computer Engineering (IJECE), vol/issue: 6(1), pp. 130-138, 2016.

[21] Nurulnajah M. Z. and Ab Al-Hadi Ab Rahman, "VLSI Design of a Fast Pipelined 8x8 Discrete Cosine Transform," International Journal of Electrical and Computer Engineering (IJECE), vol/issue: 7(3), pp. 1430-1435, 2017.

[22] A. Sulong, et al., "Single Channel Speech Enhancement using Wiener Filter and Compressive Sensing," International Journal of Electrical and Computer Engineering (IJECE), vol/issue: 7(4), pp. 1941-1951, 2017.

[23] A. Sahoo and P. Das, "Dictionary based Image Compression via Sparse Representation," International Journal of Electrical and Computer Engineering (IJECE), vol/issue: 7(4), pp. 1964-1972, 2017. 
[24] D. Romero, et al., "Compressive Covariance Sensing: Structure-based compressive sensing beyond sparsity,” in IEEE Signal Processing Magazine, vol/issue: 33(1), pp. 78-93, 2016.

[25] F. Thakkar and V. K. Srivastava, "Improved compressive sensing for grayscalelmages with zigzag scanning and block DCT," Computer, Communication and Control (IC4) International Conference, Indore, pp. 1-4, 2015.

[26] H. Rueda, et al., "RGB detectors on compressive snapshot multi-spectral imagers," IEEE Global Conference on Signal and Information Processing (GlobalSIP), Orlando, FL, pp. 388-392, 2015.

[27] J. Yuan, et al., "An improved algorithm of search for compressive sensing image recovery based on lp norm," Chinese Automation Congress (CAC), Wuhan, pp. 1962-1968, 2015.

[28] A. Pramanik and S. P. Maity, "DPCM-quantized block-based compressed sensing of images using Robbins Monro approach,” IEEE International WIE Conference on Electrical and Computer Engineering (WIECON-ECE), Dhaka, pp. 18-21, 2015.

[29] A. Maronidis, et al., "Scalable image annotation using a product compressive sampling approach,” Data Science and Advanced Analytics (DSAA) IEEE International Conference, Paris, pp. 1-10, 2015.

[30] T. Wang, et al., "Depth map coding based on adaptive block compressive sensing," Signal and Information Processing (ChinaSIP), IEEE China Summit and International Conference, Chengdu, pp. 492-495, 2015.

[31] Lakshminarayana M. and M. Sarvagya, "Scaling the Effectiveness of Existing Compressive Sensing in Multimedia Contents,” International Journal of Computer Applications, vol/issue: 115(9), pp. 16-26, 2015.

[32] Lakshminarayana M. and M. Sarvagya, "Lossless Compression of Medical Image to Overcome Network Congestion Constraints," Springer, Proceedings of Third International Conference on Emerging Research in Computing, Information, Communication and Application (ERCICA-2015), Bangalore, vol. 01, pp. 305-311, 2015.

[33] M. Lakshminarayana and M. Sarvagya, "Random sample measurement and reconstruction of medical image signal using Compressive Sensing," International Conference on Computing and Network Communications (CoCoNet), Trivandrum, pp. 255-262, 2015.

[34] Lakshminarayana M. and M. Sarvagya, “Algorithm to Balance Compression and Signal Quality using Novel Compressive Sensing in Medical Images," Springer-Software Engineering Perspectives and Application in Intelligent Systems, pp. 317-327, 2016.

[35] Lakshminarayana M. and M. Sarvagya, "HAMIC: A Novel Modelling of Hybrid Algorithm for Medical Image Compression,” IEEE-INDIACom-2017, Bharati Vidyapeeth, New Delhi, 2017.

[36] Lakshminarayana M. and M. Sarvagya, "CARIC: A Novel Modeling of Combinatorial Approach for Radiological Image Compression,” SPRINGER-CSoC-2017, Tomas Bata University in Zlin, Czech Republic, 2017.

[37] Lakshminarayana M. and M. Sarvagya, "RM2IC: Performance Analysis of Region based Mixed-mode Medical Image Compression,” International Journal of Image, Graphics and Signal Processing (IJIGSP), vol/issue: 9(10), pp. 12-21, 2017.

[38] "Finding Articles, Databases and Images," Cornell University Library, 2016. https://www.library.cornell.edu/research/introduction/articles.

\section{BIOGRAPHIES OF AUTHORS}

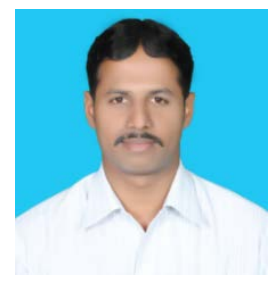

Lakshminarayana. M received the B.E. degree in Medical Electronics from Dr. AIT, Bangalore in 2005 and the M. Tech degree in Electronics from Sir. MVIT, Bangalore in 2007 under Visvesvaraya Technological University, Belgaum, where he is currently working towards the Ph.D. degree under VTU in the field of Multimedia Communication applications. He is the member of Professional bodies IEEE, MISTE, IAENG. His current research interests include the areas of Multimedia communication, Image and video processing and DSP applications in embedded system design.

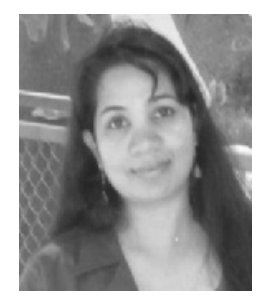

Dr. Mrinal Sarvagya, Professor, School of Electronics and Communication Engineering PhD degree in Wireless Communication from IIT Kharagpur, MTech degree in digital Communication, (Course work from IIT kanpur) from DAVV Indore and B.E degree in Electronics and communication Engineering ” from Govt Engineering College Ujjain. ”. She received the best thesis award from IIT Kharagpur in the year 2009 for her thesis titled "QoS based packet scheduling and resource allocation schemes for WCDMA UMTS”. She is the member of Professional bodies WIE, IEEE. He/She has 15 years of teaching experience, teaching various subjects like Wireless communication, Advanced Digital Communication, Computer communication and networking, real time Operating Systems, Adhoc wireless networks, Protocol Engineering. Her area of research is Wireless communication, channel equalization in OFDM-IDMA / SCM receivers, Cognitive Radio networks. Principal Investigator, VGST Karnataka "Zero padding OFDM signals for Cognitive radio Networks" (2012 -2014). Principal Investigator, IEEE Hyderabad "Multimedia Communication over Fibre Optical link” (2012) Installed at Birla science Museum Hyderabad. Currently Principal Investigator for the sponsored Project from Naval research Board, Ministry of defence India (2015).

Int J Elec \& Comp Eng, Vol. 8, No. 5, October 2018 : 2818 - 2828 